\title{
Comparison Study of Sensitivity Factors of Elements in Glow Discharge- \& Inductively Coupled Plasma- Mass Spectrometry
}

\author{
Young-Sang Kim, ${ }^{\star}$ M. Plotnikov, ${ }^{\dagger}$ and Volker Hoffmann ${ }^{\dagger}$ \\ Department of Advanced Material Chemistry, Korea University, Jochiwon 339-700, Korea. "E-mail: yskim41@korea.ac.kr \\ ${ }^{\dagger}$ Leibnitz-Institute for Solid State and Materials Research (IFW), Helmholtzstrasse 20, Dresden 01069, Germany \\ Received August 2, 2005
}

\begin{abstract}
Sensitivity factors of elements by a glow discharge mass spectrometry (GD-MS) were intensively investigated and compared with a laser ablation inductively coupled plasma-mass spectrometry (ICP-MS). In case of copper matrix, the sensitivity factor by GD-MS generally decreases with the increase of the mass number of element. The details are a little different between each data measured by Faraday and multiplier detectors. The factor by a multiplier detector drastically decreases with the mass increase in the region of low mass as in Faraday detector's case, but slowly in the high mass region. On the contrast, the sensitivity factor of solution standard by a conventional ICP-MS slowly increases with the increase of elemental mass number even though there are some exceptions such as gold and also the sensitivity factor by a laser ablation ICP-MS generally increases with mass number of element in the specimen of glass type. In case of steel matrix, any definite trends could not be shown in the relationship between the GD-MS's sensitivity factor and elemental mass.
\end{abstract}

Key Words : Glow discharge mass spectrometry, Laser ablation ICP-MS, Sensitivity factor

\section{Introduction}

In this work, sensitivity factors of elements by glow discharge mass spectrometry (GD-MS) were intensively investigated and compared with a laser ablation inductively coupled plasma-mass spectrometry (ICP-MS). Many atomic spectroscopic analysts are much interested in the relative sensitivity factor (RSF) because of the possibility to analyse samples without standard samples. Therefore, they also have tried to obtain more exact RSF values theoretically as well as experimentally.

Huneke et al. ${ }^{1}$ obtained experimental and theoretical RSF values. Many kinds of standards of $\mathrm{Al}, \mathrm{Ti}, \mathrm{Fe}, \mathrm{Ni}, \mathrm{Cu}$ and $\mathrm{Pt}$ matrix were used for the experimental RSF. The result of a VG9000 GD-MS was used for that as well. The RSF was independent on the matrix within the experimental errors. At the same time, theoretical RSF values also were calculated using their equations in which ion yield by GD source, ion transmission through transfer lens system and mass analysing and detection efficiencies were included. The ion yield was influenced by several processes such as a sputtering, transport phenomena, ionisation, recombination, and ion extraction in the source. The very good agreement was obtained in the most cases with some exceptions in elements of high ionisation potential.

Following equation was used for the calculation of theoretical values:

$$
\left(\frac{I_{s}}{I_{s}}\right)=a\left(\frac{c_{x}}{c_{s}}\right)+b
$$

where I: intensity, $c$ : atomic concentration, and subscripts $x$ and $s$ mean analyte and internal standard elements respectively. Equation (1) could be rearranged to

$$
\left(\frac{c_{x}}{c_{s}}\right)=\operatorname{RSF}\left(\frac{x}{S}\right) \times\left(\frac{I_{x}}{I_{x}}\right)
$$

Here, $R S F(x / s)$ is the inverse of slope $a$ in eq. (1), $c$ is expressed in the weight composition.

Besides, Bogaerts et al..$^{2-8}$ developed a model to explain the experimental RSF in GD-MS based on transport and ionisation of sputtered atoms by using the densities and flux energy distributions of the plasma species. The densities and distributions were obtained from explicit mathematical modelling work of dc-GD. It was found that the model based on transport and Penning ionisation only was not able to explain the variation in experimental RSFs for all elements. The correlation with the availability of suitable energy levels for charge transfer of the elemental ions was concluded to strongly suggest that ionisation by an asymmetric charge transfer between argon ions and the sputtered atoms could be used to explain the variations in the RSF values.

In addition to such investigations, many analysts have concentrated their efforts on the improvement of the sensitivity of GD-MS, and their understanding of the RSFs was very essential for this work.

On the other hand, RSF has been advanced in ICP-MS recently. Ramendik et al. ${ }^{9}$ determined RSFs of 68 elements by ICP-MS and roughly described ionisation processes in ICP with Saha equation. The absolute electro-negativity of atoms was found to substantially affect the accuracy of RSFs calculation for chemically active elements. The mean relative systematic error of RSFs could be improved to 0.30 by using the absolute electro-negativity. Oksenoid and Ramendik ${ }^{10}$ used an analytical approach to investigate the complicate plasma processes. The approach consisted of the use of fitting parameters and determination of correlation of RSFs with the main physical-chemical properties of analytes. The 
multi-element semi-quantitative analysis without standards can be developed by this approach.

In our laboratory, an interesting tendency in sensitivity factors of elements by GD-MS and the laser ablation ICPMS was observed. That is, the tendency was oppositely shown between GD-MS and the ICP-MS and the former works about RSF should be completed as well. Therefore, this work was a start to obtain useful data and sensitivity factors of elements in copper and steel standards by GD-MS and to compare them with sensitivity factors of the laser ablation ICP-MS.

\section{Experimental Section}

Instrument. Mainly GD-mass spectrometer and ICPmass spectrometer were used for this work as follows:

Turner Scientific TS-Sola GD-MS:

Quadrupole mass analyzer

Lab. made Grimm type source ( $8 \mathrm{~mm}$ anode)

Faraday \& electron multiplier detectors

VG PQ2 laser ablation ICP-MS

Quadrupole mass analyzer

ICP (27 MHz) \& Laser ablation (266 nm)

Electron multiplier detector with counting and analog modes

All of the operating conditions were optimised for the measurement of ion intensities.

Standards. Copper standards of ASMW CuI (01), CuII (02), CuIII (03), CuVI (031), CuVII (0111), CuVIII (0211), CuIX (0311) and $\mathrm{CuX}(0001)$ and steel standards of NIST 1761-67 were used for GD-MS measurement. On the other hand, the standard solutions of $10,25,100 \mu \mathrm{g} / \mathrm{mL}$ of 19 elements and glass type standards of 62 elements of given concentrations were used for the laser ablation ICP-MS. Every measurement has been done under optimised conditions and the measured intensities were treated with developed software to convert them to the corrected intensities.

\section{ICP-MS}

At first, calibration curves by ICP-MS should be made for each element to obtain the sensitivity factor from the slope. As an example, the calibration curve of uranium 238 in a liquid solution was shown in Figure 1. Experimentally

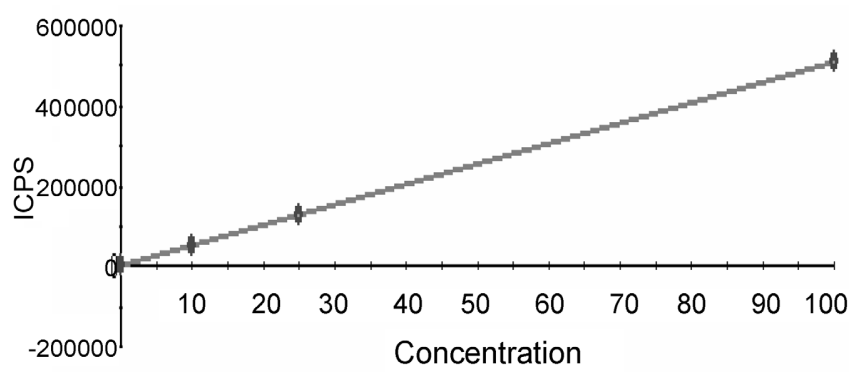

Figure 1. Calibration curve of U238 in liquid solution by a laser ablation ICP-MS.

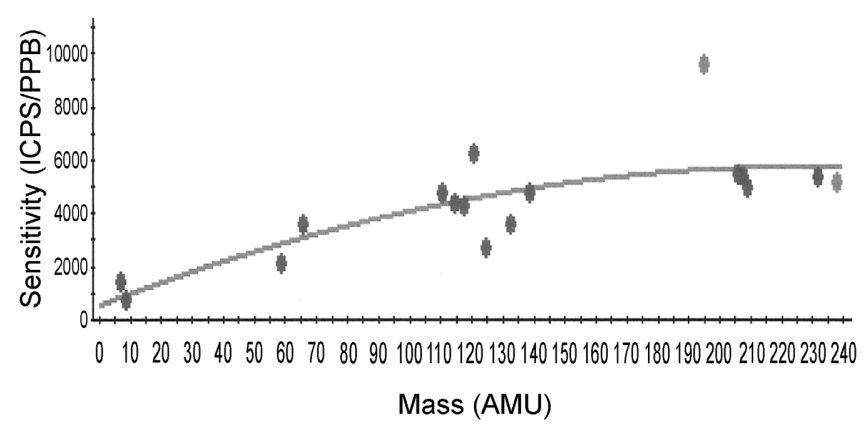

Figure 2. Sensitivity factors of elements in liquid solution by ICPMS.

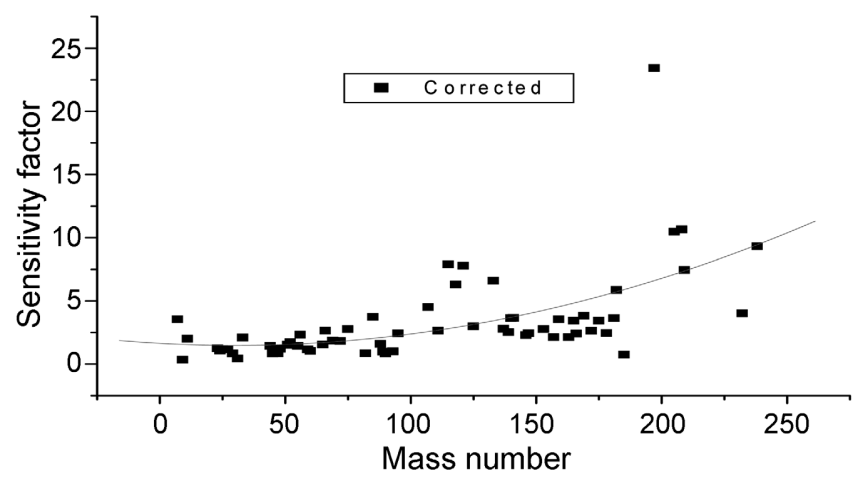

Figure 3. Sensitivity factors of elements in glass type specimen by a laser ablation ICP-MS.

measured intensity was plotted against the concentration of $\mathrm{U}$ in aqueous solutions. In order to make such graphs, three standard solutions of 10,25 and $100 \mu \mathrm{g} / \mathrm{mL}$ were prepared in $2 \% \mathrm{HNO}_{3}$ for 19 elements from $\mathrm{Li}$ to $\mathrm{U}$.

On the other hand, glass type specimens of 62 elements were obtained from NIST 610 and 612 . The intensity was measured under the optimized instrumental conditions. The regression equation for the calibration curves was follow:

$$
I_{x}=\text { slope } \cdot c_{\text {certified }}+b
$$

where, $c_{\text {certified }}$ is in mass $\%$.

After the slope values were picked up from such calibration curves, the values were converted to sensitivity factors by the correction with following equation:

$$
S F=\frac{\text { Slope } \times 100}{\% \text { Abundance } \times I D}
$$

where $\%$ abundance is for measured isotope, $I D$ is the ionisation degree of elements. The ionization degree in the plasma was calculated with the Saha equation.

Sensitivity factors were plotted versus the mass number of elements as in Figure 2 and 3. The sensitivity factor increases with the increasing of atomic mass in the graph of liquid solution. But osmium and gold have too high values of $S F 82629$ and $S F 21759$ to be scale-over of this graph so that the values were omitted in this graph. In spite of high ionization potentials, they showed much high sensitivity factors here. These high factors could be considered to be due to other factors more predominantly than ionization 

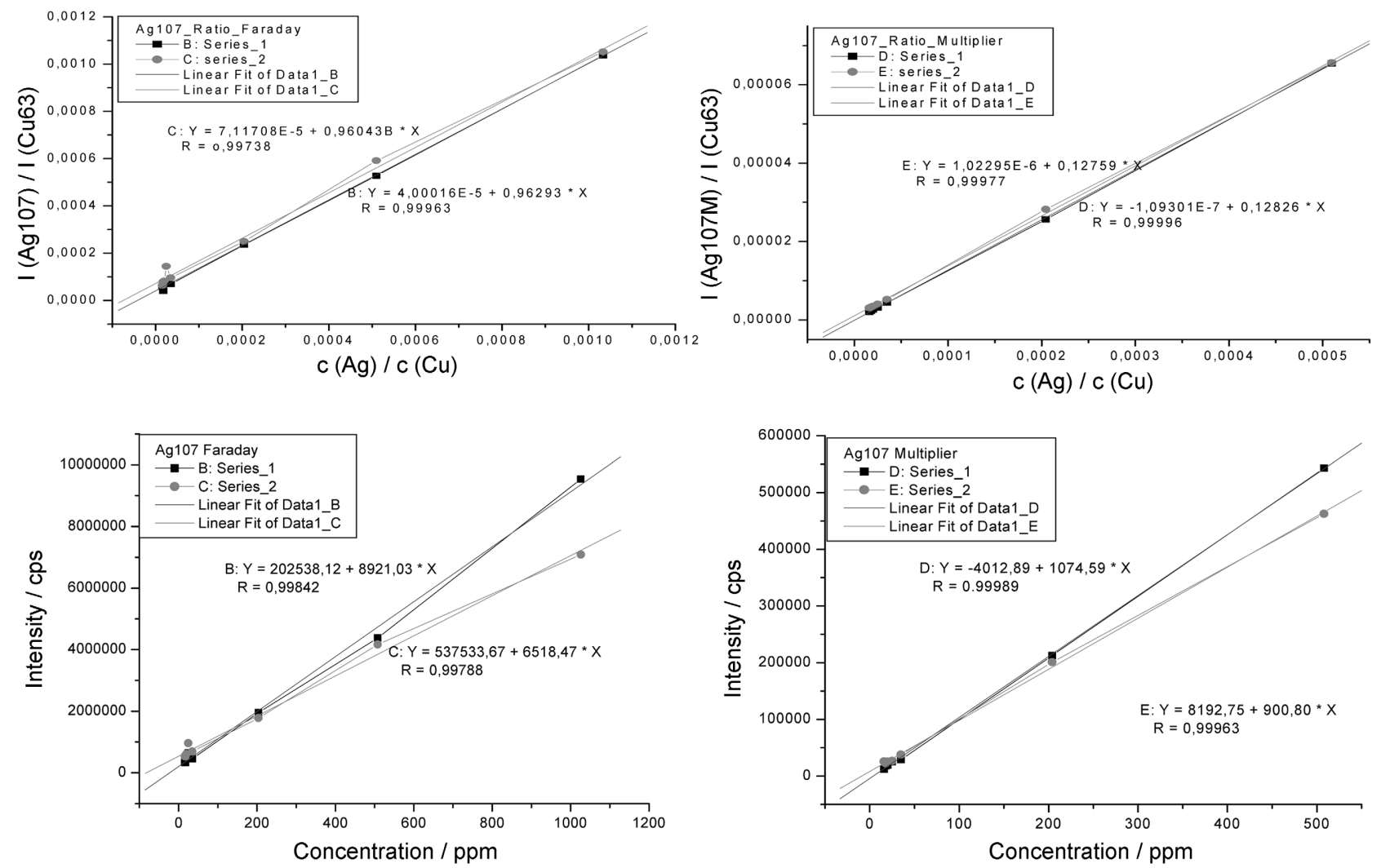

Figure 4. GD-MS calibration curves of Ag107 in copper standards by the Faraday cup detector and the electron multiplier detector.

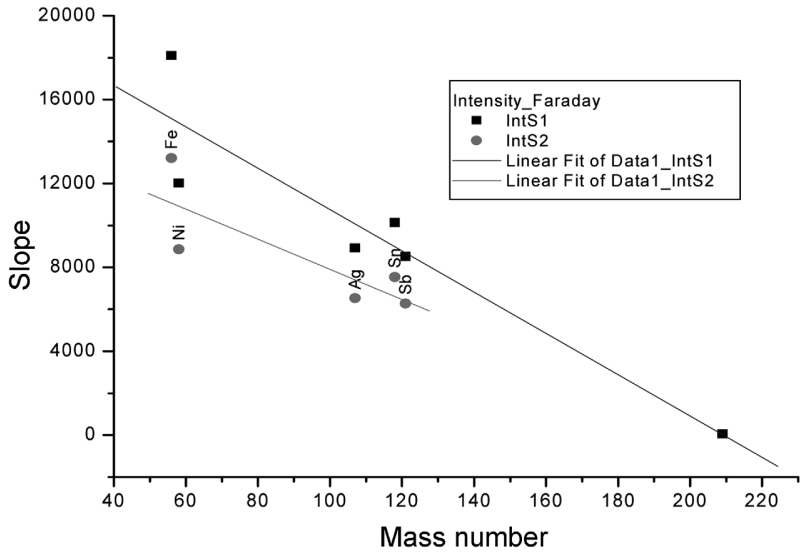

Mass dependency on sensitivity

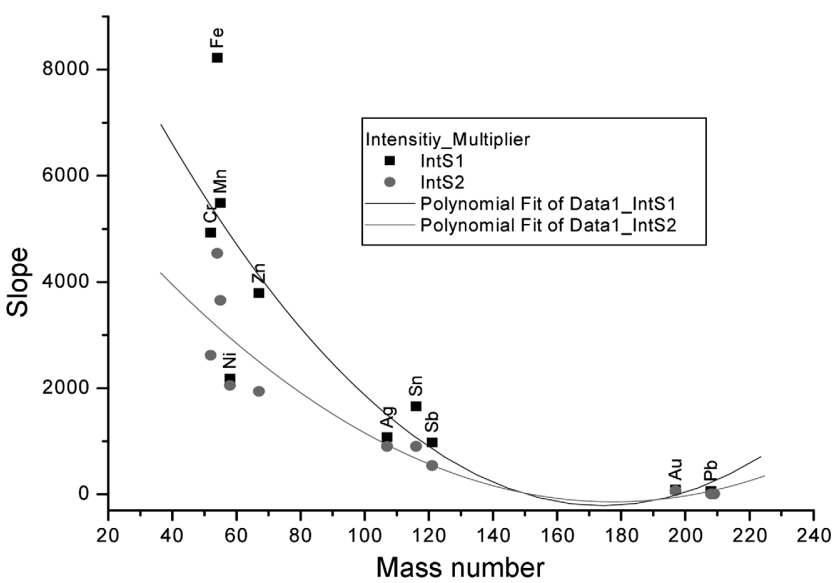

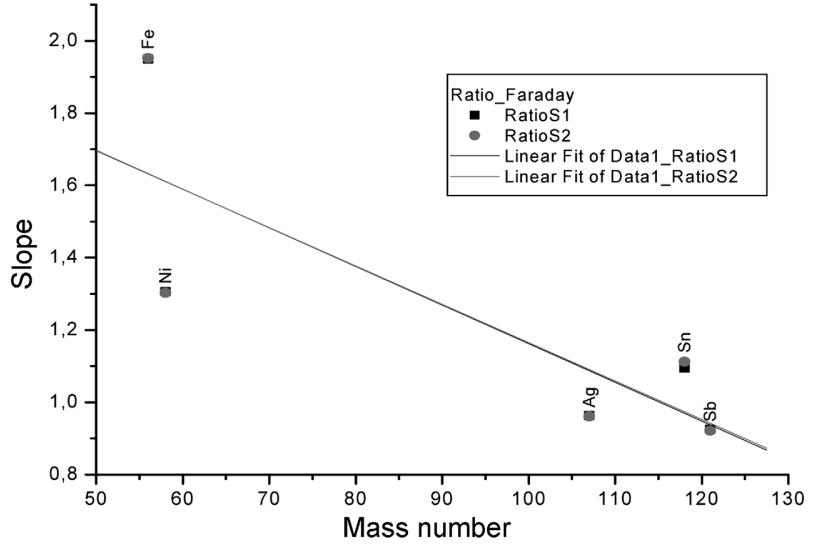

Mass dependency on sensitivity

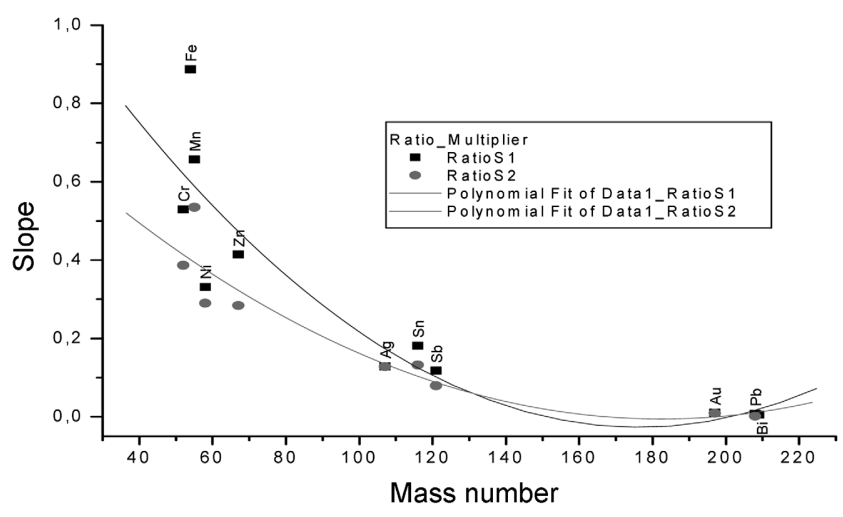

Figure 5. Mass dependence on sensitivity factor in Cu standards by GD-MS. 

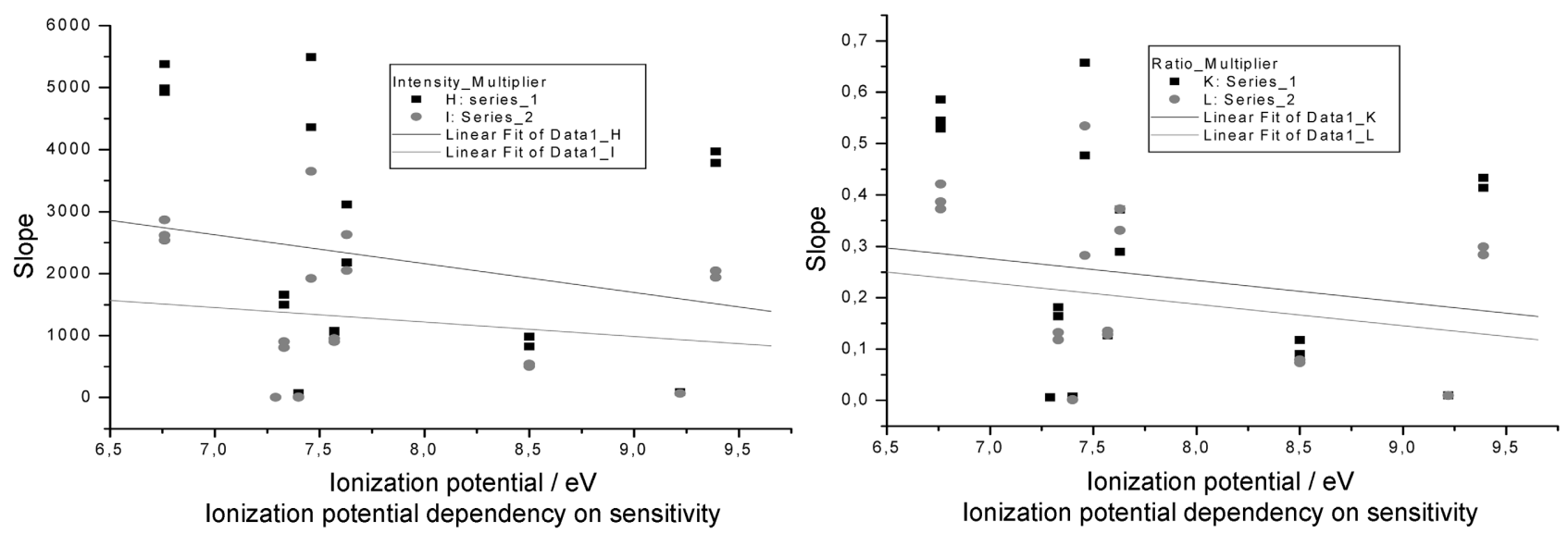

Figure 6. Dependence of an ionization potential on the variation of the sensitivity factor of element by GD-MS.

potential or the correction of the ionisation degree by the Saha equation did not work satisfactorily.

Also in Figure 3, it was shown that the sensitivity factor slowly increases with the increase of elemental mass number even though there are several exceptions such as gold. In the laser ablation ICP-MS, the sensitivity factor generally
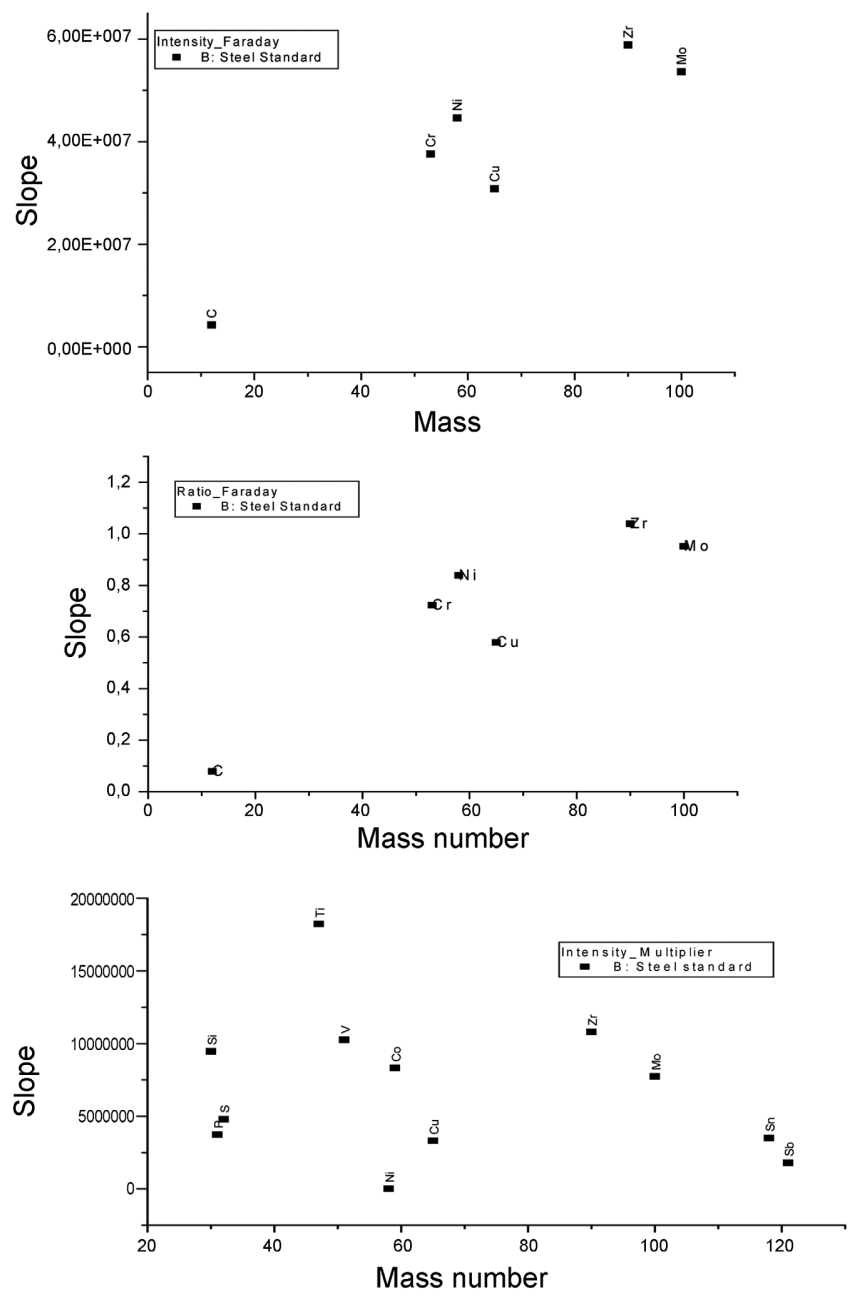

Figure 7. Mass dependence on sensitivity factor in steel standards by GD-MS. increases with mass number of element in both types of specimens. In Huneke's investigation, also abnormally high sensitivity factors were given for the elements of high ionisation potential such as $\mathrm{Be}(9.3 \mathrm{eV}), \mathrm{C}(11.2 \mathrm{eV}), \mathrm{Zn}(9.3$ $\mathrm{eV})$ and $\mathrm{Cd}(8.99 \mathrm{eV})$.

\section{GD-MS}

Copper standards. At first, 4 graphs were plotted to show calibration curves for silver of mass number 107 in copper standards as in Figure 4. The intensities of elements were measured simultaneously with Faraday cup as well as electron multiplier detectors. Argon was used as a plasma gas for this measurement. And the intensities were corrected on the isotope abundance with the term, $\left(I_{x} \cdot 100 /\right.$ Abundance), of following equation:

$$
\frac{I_{x} \cdot 100}{\text { Abundance }}=S F \cdot c_{\text {certified }}+b
$$

Here, $c_{\text {certified }}$ is in mass $\%$.

And then two kinds of graphs were made by using intensity and intensity ratio for each isotope. The curves were made by plotting the intensity versus concentration and by plotting the intensity ratio of $\mathrm{Ag} 107$ to $\mathrm{Cu} 63$ versus the concentration ratio of $\mathrm{Ag}$ to $\mathrm{Cu}$. Data of both of Faraday cup and secondary electron multiplier detectors were used to measure the intensities for these graphs. In fact, the intensity was measured with the same standards two times at different days under the same conditions. Therefore two series of data were used for each curve. Different intensities of the same samples were measured at different days even though the same conditions are used. The slope values were used as sensitivity factors of each element. The ratio could compensate any changes in the sputtering rate, which obviously do not only exist in the $\mathrm{Cu}$ and $\mathrm{Fe}$ standard set, but also are suitable to correct for linear instrument drifts.

In order to see the relationship between the sensitivity factor and mass number at GD-MS, the sensitivity factors were plotted versus the mass number of element. Also four graphs were made with four kinds of sensitivity data from the calibration curves of Figure 4. The general trend was that 
the sensitivity factor generally decreases with the increase of the mass number of element as in Figure 5. But the details are a little different between Faraday and multiplier detectors. In case of multiplier detector, the factor drastically decreases with the mass increase in the low mass region as in Faraday detector's case, but slowly in the high mass region.

As shown in ICP-MS, the sensitivity factor of Fe in all four graphs is much higher compared to neighboring elements. The cause is not known exactly, but it seems to be due to other factors such as itself property. Whereas in the case of the Faraday cup detector the ratio compensates the instrument drift between the two series, this was not done in the case of the multiplier.

In addition, the dependence of the ionization potential on the variation of sensitivity factor of element was investigated because it can predominantly influence the ion production in plasma. Therefore, sensitivity factors from intensity and intensity ratio curves were plotted against the ionization potential of element (Figure 6). Two graphs by the multiplier detector were shown here because many data could not be obtained by the Faraday detector in the low concentration area. From these graphs, any definite trends could not be found. Any definite trends were also not found in LA-ICPMS data, if the data were not grouped in one period of the periodic table of elements.

Steel standards. As in the case of copper standards, mass dependency on the sensitivity factors was investigated using the data of steel standards. At first, calibration curves were prepared for 14 elements using intensities and intensity ratios. The slope values were picked up as sensitivity factors and the factors were plotted versus the mass number of element (Figure 7). But any definite trends could not be observed in this case. The carbon of high ionization potential $(11.26 \mathrm{eV})$ showed a much lower sensitivity factor as expected.

In summary, firstly it was shown that SF increased with increasing of mass number for both specimens of liquid solution and glass type plate in laser ablation ICP-MS. This is considered to be an instrumental function. On the other hand, SF by GD-MS approximately decreased with increasing of mass number and a definite tendency could be not shown between $\mathrm{SF}$ and ionization potential in $\mathrm{Cu}$ standards. The instrument drift can be compensated by the use of relative SF with the exception of the drift of the multiplier. In case of steel standards, any definite trends could be not observed in the relationship between SF and elemental mass.

\section{References}

1. Vieth, W.; Huneke, J. C. Spectrochim. Acta 1991, 46B(2), 137.

2. Bogaerts, A.; van Straaten, M.; Gijbels, R. Spectrochim. Acta 1995, 50B, 179.

3. Bogaerts, A.; van Straaten, M.; Gijbels, R. J. Appl. Phys. 1995, 77, 1868.

4. Bogaerts, A.; Gijbels, R.; Goedheer, W. J. J. Appl. Phys. 1995, 78, 2233.

5. Bogaerts, A.; Gijbels, R. Phys. Rev. A 1995, 52, 3743.

6. Bogaerts, A.; Gijbels, R. J. Appl. Phys. 1995, 78, 6427.

7. Bogaerts, A.; Gijbels, R. J. Appl. Phys. 1996, 79, 1279.

8. Bogaerts, A.; Gijbels, R. J. Anal. Atomic Spectrom. 1996, 11(9), 841.

9. Ramendik, G. I.; Fatjushina, E. V.; Stepanov, A. I.; Sevastyanov, V. S. Fresenius J. Anal. Chem. 2000, 368, 166.

10. Oksenoid, K. G.; Ramendik, G. I. J. Anal. Chem. USSR 1996, 51, 83; tranfl. from Zh. Anal. Khim. 1996, 44, 2157. 